\title{
Optimising the interconnection of free-space to fibre quantum networks
}

\author{
Alejandro Ocampos-Guillén ${ }^{1}$, Natalia Denisenko $^{1}$, and Verónica Fernández-Mármol ${ }^{1, *}$ \\ ${ }^{1}$ Spanish National Research Council (CSIC), Institute of Physical and Information Technologies \\ (ITEFI), Serrano 144, 28006 Madrid, Spain.
}

\begin{abstract}
Optimising the interconnection between free-space and fibre links will be necessary for future quantum communication networks. In daylight free-space quantum communication based on direct detection, the required Field Of View (FoV) of the receiver system needs to be minimised to reduce solar background noise coupling into the detectors. Reducing the FoV requires minimising beam wander effects caused by atmospheric turbulence through active optics. We implement a fine tracking system designed to correct tip and tilt wavefront aberrations, using two feedback loops; each of them consisting of a quadrant detector and a fast steering mirror for stabilising the beam in the whole optical axis of the receiver. We test the performance of the tracking system with different optical fibres in order to evaluate the reduction in the quantum bit error rate (QBER) caused by solar background noise. A reduction of $75 \%$ for single mode fibre was obtained, and $45 \%$ reduction for a $25 \mu \mathrm{m}$ core diameter fibre, both cases for strong turbulence $\left(C_{n}^{2} \sim 10^{-12}-10^{-13} \mathrm{~m}^{-2 / 3}\right)$ and $100 \mathrm{~m}$ propagating channel. These results look promising for enabling free-space Quantum Key Distribution $(\mathrm{QKD})$ in wireless networks for realistic/adverse conditions such as daylight and strong turbulent regimes.
\end{abstract}

\section{Introduction}

Quantum safe communications is now recommended by national securities agencies worldwide. Quantum Key Distribution (QKD) is a quantum safe cryptographic primitive that ensures key transmission with information-theoretic security [1, 2].

Due to limitations in the maximum distance of fibre links caused by absorption in optical fibres, free-space links are essential to increase the range of quantum networks to a global scale. Many advances have been reported in recent years from terrestrial stationary links of a few kilometres [3-5], to satellite to ground links [6-9]. Tests on mobile terrestrial links using drones and UAVs are also being developed [10-13].

The Quantum Bit Error Rate (QBER) of a QKD link depends on both the channel and receiver losses, and on the noise of the system, which in the case of direct detection-based QKD systems is especially affected by solar background noise that couples into the receiver's detectors.

${ }^{*}$ Corresponding author: veronica.fernandez@iec.csic.es 
Therefore, it is vital to reduce this noise, which can be achieved by reducing the Field of View (FoV) of the receiver through using small core optical fibres that collect and guide the quantum signal to the detectors in the receiver. However, this must be performed in conjunction with active beam tracking techniques to avoid signal losses. Tracking techniques reduce the spot focal wander at the desired optical plane of the receiver caused by atmospheric turbulence.

In this work, we test a fine tracking system designed for improving the free-space to fibre interface for QKD systems, in high turbulence regimes as those typically observed in urban areas in daylight. The system compensates for tip and tilt aberrations of the wavefront using two Quadrant Detectors (QD), each connected to a Fast Steering Mirror (FSM). A double loop system provides with interesting advantages, such as stabilisation in the whole optical axis of the receiver, facilitating the optical design and layout. On the other hand, using QDs instead of high frame rate CCD/CMOS cameras for high speed correction of atmospheric effects, benefit from a low cost and compact design, which makes them suitable for integration in mobile and lightweight platforms; a foreseeable application of mass implementation. Cameras also require more complex postprocessing, which may increase the costs of assisting hardware, and the size of the whole system.

The paper is organized as follows: section 2 introduces the experimental set up implemented to test the tracking system; section 3 briefly describes how the characterization of the turbulence conditions is performed; section 4 shows the experimental results obtained in different days, and both the estimated reduction in QBER and increase in the Secret Key Rate (SKR).

\section{Experimental set up}

Since our study focuses in analysing the performance of a tracking system for a QKD link, the QKD emitter was approximated by a single diode laser source connected to a beam collimator emitting a $10 \mathrm{~mm}$ diameter beam.

On the other hand, the receiver is composed of a Schmidt-Cassegrain telescope for focusing the beam with minimal aberrations. A 50/50 beamsplitter then divides the beam in two paths: one for tracking purposes with two QDs, each in a feedback loop to a FSM, and another path composed of a lateral effect PSD and an optical fibre for observing the correction. Both correcting systems are in closed-loop, which have been proven to perform better than the open-loop strategy [14].

A schematic representation of the system is shown in Figure 1.

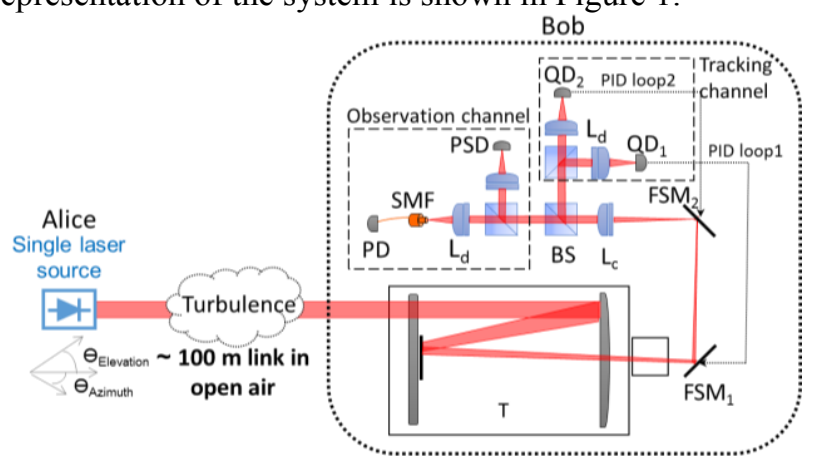

Fig. 1. Scheme of the experimental system. PSD: lateral-effect position sensitive detector; QD: quadrant detector; BS: beamsplitter; FSM: fast steering mirror; $\mathrm{L}_{\mathrm{c}}$ : collimating lens; $\mathrm{L}_{\mathrm{d}}$ : detector lens; T: Schmidt-Cassegrain telescope. 
Each FSM is connected to a QD through a Proportional Integrative Derivative (PID) control loop implemented electronically by hardware, which processes the received signal from the detectors and sends instructions to the FSM. The mirrors rotate a certain angle at a maximum frequency of around $500 \mathrm{~Hz}$ to compensate for the tip and tilt deviations of the beam.

We use two wavelengths with good transmission in the atmosphere for comparison purposes to test the tracking system: a visible $670 \mathrm{~nm}$ and an infrared $850 \mathrm{~nm}$. The former is practical to facilitate the optical alignment, performed manually, and the latter is more commonly used in quantum communication for its well transmittance in the atmosphere and compatibility with commercially available Silicon single photon detectors.

The link is located in a metropolitan environment (inside the city of Madrid, Spain), where the emitter and the receiver are placed in the ITEFI-CSIC building, facing each other and separated by $30 \mathrm{~m}$. We use two reflecting mirrors to achieve a $100 \mathrm{~m}$ link (Figure 2).

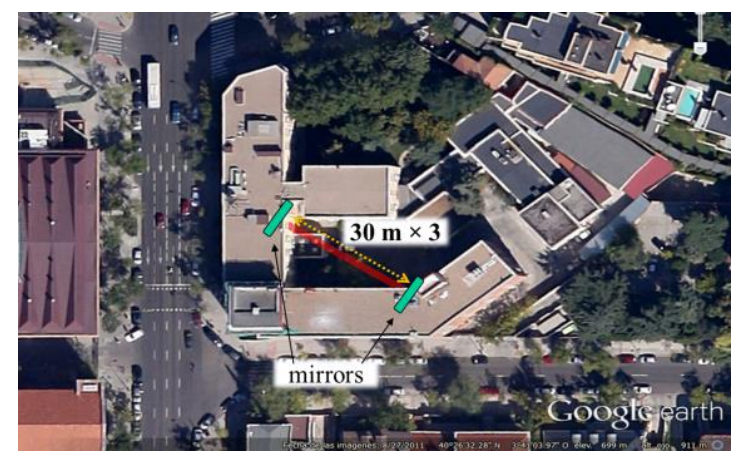

Fig. 2. View of the ITEFI-CSIC building (coordinates: $40^{\circ} 26^{\prime} 32.5^{\prime \prime} \mathrm{N} 3^{\circ} 41^{\prime} 08.2^{\prime \prime} \mathrm{W}$ ) and the free-space link. Source: Google Earth.

\section{Characterization of the turbulent regime}

For characterizing the performance of the system, we measure the deviation of the beam centroid at the focal plane of the receiver, also known as spot focal wander, as well as the power coupled into the fibres. With those results, we estimate the reduction in the Quantum Bit Error Rate (QBER) for determining the potential of the system in QKD links.

We estimate the turbulent regime through the refractive index structure parameter $C_{n}^{2}$. We obtain its value from the beam propagation theory in free-space, according to Kolmogorov's theory of turbulence [15].

A scheme of the tip and tilt aberration effects is shown in Figure 3.

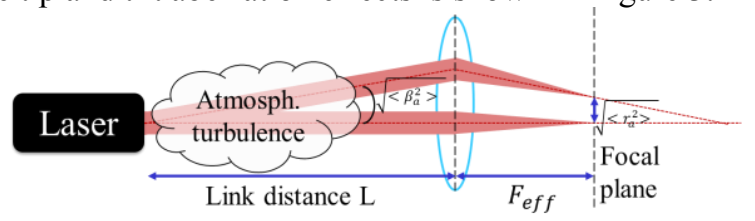

Fig. 3. Scheme of the beam wander effects over a laser traveling through the atmosphere and being collected by a lens.

We quantify those effects by measuring the root-mean-square $(\mathrm{rms})$ of the deviations of the focal spot position $\left(\sqrt{\left\langle r_{a}^{2}\right\rangle}\right)$. Later, we substitute this value to calculate the angle of arrival $\sqrt{\left\langle\beta_{a}^{2}\right\rangle}$, and subsequently $C_{n}^{2}$ through the following equations: 


$$
\begin{aligned}
& \sqrt{\left\langle\beta_{a}^{2}\right\rangle}=\operatorname{atan}\left(\frac{\sqrt{\left\langle r_{a}^{2}\right\rangle}}{F_{e f f}}\right) \\
& C_{n}^{2}=\frac{\left\langle\beta_{a}^{2}\right\rangle}{2.91 L\left(2 \omega_{G}\right)^{-1 / 3}}
\end{aligned}
$$

Where $F_{e f f}$ is the effective focal length of the system, $L$ is the link distance and $2 \omega_{G}$ is the diameter of the receiver aperture at the receiver.

Strong turbulence regimes are considered for $C_{n}^{2}$ of $\sim 10^{-13} \mathrm{~m}^{-2 / 3}$, moderate regimes for values $\sim 10^{-17} \mathrm{~m}^{-2 / 3}$, and weak turbulence regimes for lower values [15].

\section{Results}

\subsection{Tracking error}

The system shown in Figure 1 was tested for two different wavelengths separately, 670 and $850 \mathrm{~nm}$, for several days in the $100 \mathrm{~m}$ link. The values of the rms focal spot wander were measured at the PSD, calculating $C_{n}^{2}$, using equations 1 and 2 , and the tilt angle of the system, using equation 3 .

$$
\text { tilt angle }=\frac{\sqrt{\left\langle r_{a}^{2}\right\rangle}}{F_{\text {eff }}}
$$

The calculation of this angle is useful to compare the effects of beam wander with and without tracking correction (see Figure 4).

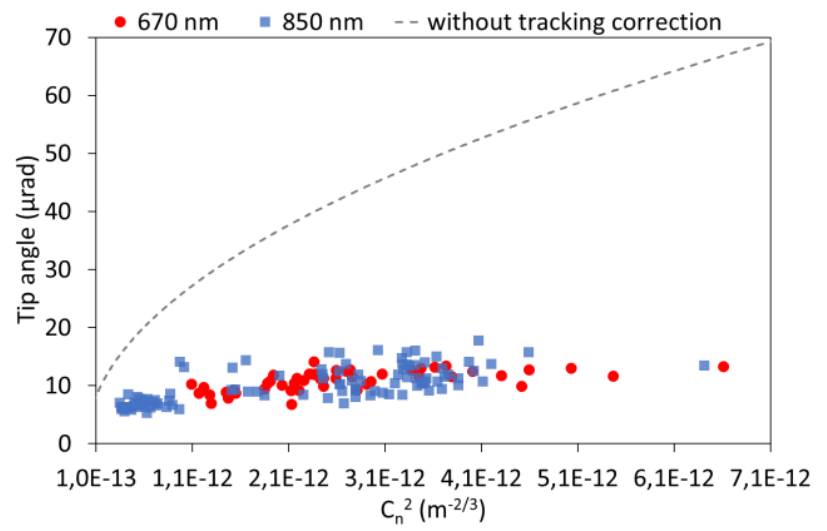

Fig. 4. Tracking error measured for several days from December of 2017 to March of 2018, using two wavelengths separately, in the $100 \mathrm{~m}$ link.

The results show that the tip angle with correction (also known as tracking error) is practically constant with turbulence strength for both wavelengths (see figure 4).

\subsection{Experimental and estimated coupling efficiency}

We estimate the coupling efficiency $\left(\eta_{C}\right)$ under weak to moderate turbulence conditions using the following equation [16]: 


$$
\eta_{C}=8 a^{2} \int_{0}^{1} \int_{0}^{1} \exp \left[-\left(a^{2}+\frac{A_{R}}{A_{C}}\right) \cdot\left(x_{1}^{2}+x_{2}^{2}\right)\right] \cdot I_{0}\left(2 \frac{A_{R}}{A_{C}} x_{1} x_{2}\right) \cdot x_{1} \cdot x_{2} d x_{1} d x_{2}
$$

Where:

- The parameter $a$ is the ratio of the receiver lens radius to the radius of the back-propagated fibre mode.

- $A_{c}$ is the spatial coherence area.

- $A_{R}$ is the area of the receiver aperture.

- $I_{0}(x)$ is the modified Bessel function.

- $x_{1}$ and $x_{2}$ are the radial integration variables of the receiver planes, normalized to the receiver lens radius.

In May of 2018 more data were measured and characterized for obtaining results in lower turbulence than that experimented in the previous studies. These data are shown in figure 5 together with the theoretical estimation given by numerically solving equation 4 .

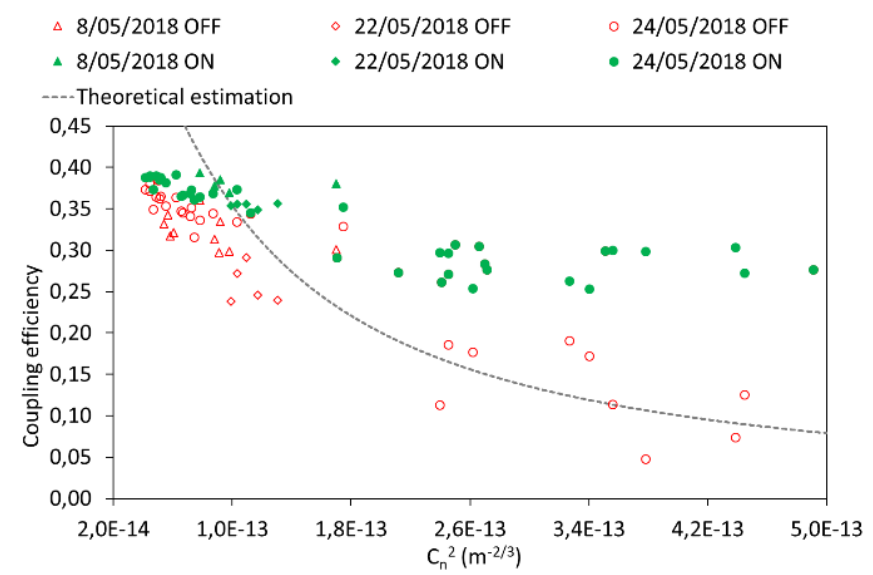

Fig. 5. Experimental coupling efficiency measured at a fibre of a core diameter of $9.5 \mu \mathrm{m}$. The data were measured along several days in May of 2018 in the $100 \mathrm{~m}$ link using a two FSMs based correcting system. The laser beam wavelength was $850 \mathrm{~nm}$. Correcting system is deactivated for data labelled as $\mathrm{OFF}$, and activated for those labelled as $\mathrm{ON}$.

The theoretical estimation is only valid for systems without tracking. The experimental results are slightly lower than the theoretical line, due to probably a small misalignment between the beam and the fibre. It is clear from the experimental data that the coupling efficiency increases when the tracking is $\mathrm{ON}$, and the degree of the improvement is larger for higher turbulent regimes since there is more range for correction (similarly to fig. 4).

\subsection{QBER reduction}

The tracking system was also tested using fibres of different diameter core, of 9.5, 25 and 50 $\mu \mathrm{m}$ separately. We measured the coupling efficiency with and without the action of the correcting system, and compared them to quantify the system performance.

Results show how the power distribution is shifted to higher values of the normalized power when the correcting system is activated, which means that the coupled optical power in the fibre is increased with the correction, as desired. 

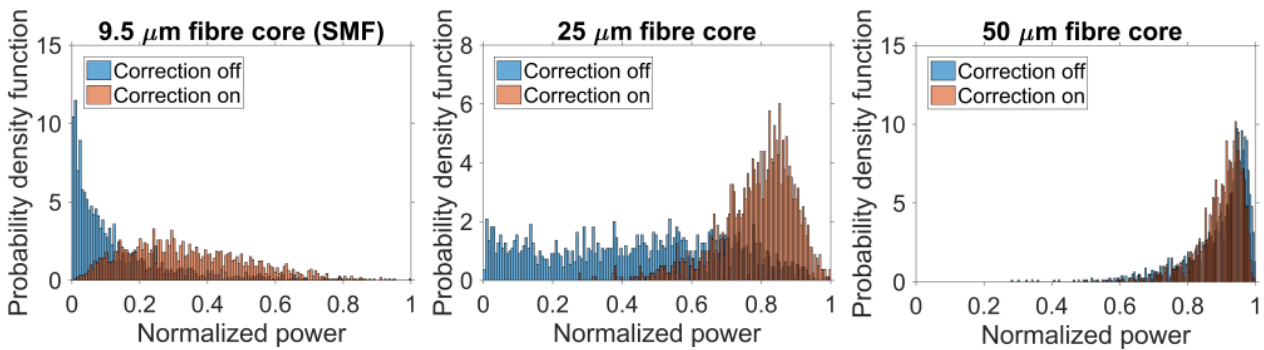

Fig. 6. Probability Density Functions (PDF) measured for 6 minutes in the $100 \mathrm{~m}$ link using the tracking system. The laser beam wavelength was $670 \mathrm{~nm}$. Turbulence conditions of $C_{n}^{2}=2 \cdot 10^{-12} \mathrm{~m}^{-2 / 3}$.

In addition, the power is shifted to higher values for larger fibres, which is also to be expected. No improvement was observed for the largest core fibre $(50 \mu \mathrm{m})$, from which it can be concluded that the beam fluctuations area without correction were equal or smaller than the fibre core area.

We measured the coupling efficiency ratio (correction OFF over correction $\mathrm{ON}$ ) and with this we estimated the QBER reduction; with QBER caused by background being:

$$
Q B E R_{b} \alpha\left(\frac{D}{2 B}\right)
$$

Where $D$ is the background photon rate, and $B$ the bit rate received by Bob.

Taking into account that the ratio in $\eta_{C}$ is the same as the ratio in $B$, the QBER reduction (\%) can be estimated by:

$$
\text { Reduction in } Q B E R(\%)=\left(1-\frac{\eta_{c_{-} o f f}}{\eta_{c_{-} o n}}\right) \cdot 100
$$

Table 1. Results of coupling efficiency and reduction in QBER due to solar background noise obtained using the $670 \mathrm{~nm}$ when the tracking system is OFF and ON, in the $100 \mathrm{~m}$ link.

\begin{tabular}{|c|c|c|c|}
\hline $\begin{array}{c}\text { Fibre core } \\
\text { diameter } \\
(\mu \mathrm{m})\end{array}$ & $\eta_{c_{-} \text {off }}$ & $\eta_{c_{\text {_on }}}$ & $\begin{array}{c}\text { Reduction in } \\
\text { QBER }\end{array}$ \\
\hline 9.5 & 0.05 & 0.19 & $75 \%$ \\
\hline 25 & 0.35 & 0.64 & $45 \%$ \\
\hline 50 & 0.81 & 0.81 & $0 \%$ \\
\hline
\end{tabular}

As can be seen in Table 1, the maximum QBER improvement is achieved using the fibre of $9.5 \mu \mathrm{m}$.

The increase in the SKR depends on the actual value of the QBER and the core fibre diameter. For the SMF $9.5 \mu \mathrm{m}$ a SKR increase of $84 \%$ and $97 \%$ for QBER values of $4.3 \%$ to $7.5 \%$, respectively, was estimated, corresponding to the lowest and highest values measured in real conditions for the system this group developed in [17]. For the $25 \mu \mathrm{m}$ fibre SKR values of $59 \%$ and $91 \%$ were obtained for the same aforementioned values of the QBER.

\section{Conclusions}

The tracking system developed in this work has been largely tested over several days to analyse its potential use in QKD links in high turbulence regimes. Considerable improvement on the coupling efficiency and reduced area of the beam fluctuations was observed for two 
typical wavelengths used in tracking and quantum communications. The developed system is a cost effective solution for implementation in wireless quantum communication networks for a variety of short distance applications, including point-to-point stationary and/or mobile platforms.

Moreover, the reduction in QBER is quite promising to enhance the performance of QKD links using either a SMF fibre or a Multi-Mode Fibre (MMF). The advantage of the SMF is that the receiver system could be attached to a commercial fibre network or standard telecommunication component and act as a relay for the quantum signal from free-space platforms to fibre networks. On the other hand, the MMF provides a higher coupling efficiency, which could be an advantage in other scenarios, such as direct key distribution to the receiver stations. In addition, with the achieved reductions in $\mathrm{QBER}_{\mathrm{b}}$ of $75 \%$ for the SMF and $45 \%$ for the MMF, QKD links in daylight become feasible.

In summary, a tracking system has been designed with cheap and off-the-shelf components, capable of increasing the robustness of QKD applications under strong turbulence regimes, with potential applications as relays between free-space platforms and metropolitan fibre networks.

We would like to thank Ministerio de Economía y Competitividad, project TEC2015-70406-R (MINECO/FEDER, UE) and Fondo Social Europeo through Programa Operativo de Empleo Juvenil and Iniciativa de Empleo Juvenil (YEI).

\section{References}

1. C. H. Bennett and G. Brassard, Quantum cryptography: Public key distribution and coin tossing, Proc. of IEEE Int. Conf. Comput, pp. 175-179 (1984).

2. Bennett, C. H. Quantum cryptography using any two nonorthogonal states. Phys. Rev. Lett. vol. 68, pp. 3121-3124 (1992).

3. M.P.J. Lavery, C. Peutinger, K. Günthner, P. Banzer, D. Elser, R.W. Boyd, M.J. Padgett, C. Marquardt and G. Leuchs, Free-space propagation of high-dimensional structured optical fields in an urban environment, Sci. Adv. vol 3, no. 10, e1700552 (2017).

4. S. Liao, H. Yong, C. Liu, G. Shentu et al., Long-distance free-space quantum key distribution in daylight towards inter-satellite communication. Nat. Phot. vol. 11, pp. $509-513$ (2017).

5. Y. Gong K. Yang, H. Yong, J. Guan, G. Shentu, C. Liu, F. Li, Y. Cao, J. Yin, S. Liao, J. Ren, Q. Zhang, C. Peng and J. Pan, Free-space quantum key distribution in urban daylight with the SPGD algorithm control of a deformable mirror, Opt. Expr. vol. 26, no. 15 , pp. $18897-18905$ (2018).

6. S. Liao, W. Cai, W. Liu, L. Zhang et al., Satellite-to-ground quantum key distribution Nat. vol. 549, pp. 43 - 47 (2017).

7. J. Ren, P. Xu, H. Yong, L. Zhang et al., Ground-to-satellite quantum teleportation, Nat. vol. 549, pp. 70 - 73 (2017).

8. S. Liao, W. Cai, J. Handsteiner, B. Liu et al., Satellite-relayed intercontinental quantum network, Phys. Rev. Let. vol. 120, pp. 030501-1 - 030501-4 (2018).

9. H. Takenaka, A. Carrasco-Casado, M. Fujiwara, M. Kitamura, M. Sasaki and MToyoshima, Satellite-to-ground quantum-limited communication using a 50-kg-class microsatellite, Nat. Phot. vol. 11, pp. 502 - 508 (2017). 
10. S. Nauerth, F. Moll, M. Rau, C. Fuchs, J. Horwath, S. Frick and H. Weinfurter, Air-toground quantum communication, Nat. Phot. vol. 7, pp. 382 - 386 (2013).

11. A. D. Hill, J. Chapman, K. Herndon, C. Chopp, D. J. Gauthier, P. Kwiat, Drone-based quantum key distribution, QCrypt 2017 Conf. (2017).

12. L. Li, R. Zhang, Z. Zhao, G. Xie, et al., High-capacity free space optical communications between a ground transmitter and a ground receiver via a uav using multiplexing of multiple orbital-angular-momentum beams, Sci. Rep. vol. 7, art. num.: 17427, pp. 1 12 (2017).

13. B. Moison, B. Erkmen, E. Keyes, T. Belt, O. Bowen, D. Brinkley, P. Csonka, M. Eglington, A. Kazmierski, N. Kim, J. Moody, T. Tu and W. Vermeer, Demonstration of free-space optical communication for long-range data links between balloons on Project Loon, Proc. of SPIE vol. 10096, pp. 100960Z-1 - 100960Z-14 (2017).

14. V. Fernandez, J. Gómez-García, A. Ocampos-Guillén, and A. Carrasco-Casado, Correction of Wavefront Tilt Caused by Atmospheric Turbulence Using Quadrant Detectors for Enabling Fast Free Space Quantum Communications in Daylight, IEEE Access vol. 6, pp. 3336 - 3345 (2018).

15. L. C. Andrews and R. L. Phillips, Laser Beam Propagation Through Random Media. SPIE (2005).

16. Y. Dikmelik and F. M. Davidson, Fibre-coupling efficiency for free space optical communication through atmospheric turbulence. App. Opt. vol. 44, No. 23, pp. $4946-$ 4952 (2005).

17. M. J. García-Martínez, N. Denisenko, D. Soto, D. Arroyo, A. B. Orue, and V. Fernandez, High-speed free-space quantum key distribution system for urban daylight applications, App. Opt. vol 52, no. 14, pp. 3311 - 3317 (2013). 